\title{
Editorial: 5G for Augmented Reality
}

\author{
Zhihan Lv ${ }^{1} \cdot$ Jaime Lloret $^{2} \cdot$ Houbing Song $^{3}$ \\ Published online: 24 January 2022 \\ (c) The Author(s), under exclusive licence to Springer Science+Business Media, LLC, part of Springer Nature 2022
}

\section{Editorial}

Tremendous efforts have been devoted to $5 \mathrm{G}$ over the last decade. Due to their success, a broad range of critical applications such as smart city, home robotics, and Augmented Reality (AR) have been made possible already, or are within reach. The 5G network as a technology inherently brings aspects such as high-speed, ultra-low latency and high bandwidth - all in a wireless communication network. 5G will especially help establish the Internet of Things as an indispensable part of our world, by laying the foundation for unleashing its full potential. It's a long way to when $5 \mathrm{G}$ becomes mainstream, but businesses need to start now with developing and reimagining services and products to leverage 5G's superior capabilities. Moreover, 5G and IoT together will also help in bringing every item on the shelf to the internet by creating digital twins for them. If the number of hardware connected devices is expected to be in the billions, the potential for ordinary consumer products with digital twins to be a part of the new Internet of Things is considerably more.

Augmented Reality is a key technology that will facilitate a major paradigm shift in the way users interact with data and has only just recently been recognized as a viable solution for solving many critical needs. Enter augmented reality (AR) technology, which can be used to visualize data from hundreds of sensors simultaneously, overlaying relevant and actionable information over your environment through a headset. 5G makes AR technology much faster, with much more data flow. With easier and more accessible use, for a variety of different functions (besides video gaming), widespread adoption seems likely. In summary, AR in

\section{Zhihan Lv}

lvzhihan@gmail.com

1 Department of Game Design, Faculty of Arts, Uppsala University, Uppsala, Sweden

2 Polytechnic University of Valencia, Valencia, Spain

3 Embry-Riddle Aeronautical University, Daytona Beach, USA the era of $5 \mathrm{G}$ is a really cool upcoming wave where the vast repositories of data will enable an AR lens into the scenarios in ways that provide near immediate insight at a level of depth unimaginable previously. This special session aims to bring the latest results over 5G and Augmented Reality technologies for various applications. It can help technicians to exchange the latest technical progresses.

Aiming at the complex background and the color image with unclear target contour as the target image to be segmented, "Application of SVM and its improved model in image segmentation" [1] first used the texture and color of the image as the feature vector, and proposed an image segmentation algorithm based on SVM. The experimental results show that the accuracy rate of the improved SVM algorithm reaches $97.263 \%$, which improves the segmentation efficiency. It is verified that the improved model proposed in this paper can effectively segment complex color images.

5G mobile communication technology has become a current research hotspot. "Energy Efficiency Optimization of NOMA IoT Communication for 5G" [2] analyzed and summarized the existing Internet of Things user grouping and power allocation algorithms under multi-carrier, proposes an improved particle swarm optimization algorithm, which is simpler than traditional solution methods. The improved particle swarm optimization algorithm is close to the optimal solution, the error is within the acceptable range, and the algorithm time is complicated.

Aiming at the problem of real-time augmented reality tracking registration of motion blurred template matching image construction model, "Real Time Augmented Reality Tracking Registration Based on Motion Blur Template Matching Image Construction Model" [3] proposed a template matching target tracking algorithm based on improved efficient second-order minimization (ESM). The simulation results show that the proposed method has good effect and superior performance in real-time augmented reality tracking registration of moving targets.

In order to facilitate the implementation, The paper "Towards an IoT enabled Tourism and Visualization Review 
on the Relevant Literature in Recent 10" [4] tries to formulate a definition of IoT enabled tourism and make a visualization review on the relevant literature to IoT enabled tourism in recent 10 years. These findings are helpful for the academic circle making further efforts on IoT enabled tourism and finally facilitating the achievement of smart tourism.

For the problems of large data volume, long calculation time, and information feedback speed in traditional virtual augmented reality-based scenes, "5G-oriented virtual augmented reality scene construction and business information flow demonstration" [5] constructed a virtual augmented reality scene based on $5 \mathrm{G}$ technology. The experimental results prove that the scene constructed in this paper has the characteristics of real-time, wide field of view, high immersion and flexible.

$5 \mathrm{G}$ network is an inevitable trend in the development of mobile communications. Mobile cloud computing is a more promising technology for 5G networks. "5G Mobile Virtual Reality Optimization Solution for Communication and Computing Integration" [6] proposed a hierarchical distributed cloud service network model and a task offloading assignment algorithm in a small cell cloud scenario. At the same time, the author proposed feedback adaptive random access strategy based on the adaptive random access model. This can ensure that the throughput rate does not decrease under heavy load conditions, and at the same time, the average access delay of the existing system is reduced. Finally, a better load effect was obtained through the experiment.

\section{References}

1. Yang A, Bai Y, Liu H et al (2021) Application of SVM and its Improved Model in Image Segmentation. Mobile Netw Appl. https://doi.org/10.1007/s11036-021-01817-2

2. Xu J, Liang Z (2021) Energy Efficiency Optimization of NOMA IoT Communication for 5G. Mobile Netw Appl. https://doi.org/ 10.1007/s11036-021-01815-4

3. Tian L, Zhou J (2021) Real Time Augmented Reality Tracking Registration Based on Motion Blur Template Matching Image Construction Model. Mobile Netw Appl. https://doi.org/10.1007/ s11036-021-01816-3

4. Guo X, Wang Y, Mao J et al (2021) Towards an IoT enabled Tourism and Visualization Review on the Relevant Literature in Recent 10 Years. Mobile Netw Appl. https://doi.org/10.1007/ s11036-021-01813-6

5. Jin A, Zhao S (2021) 5G-oriented Virtual Augmented Reality Scene Construction and Business Information Flow Demonstration. Mobile Netw Appl. https://doi.org/10.1007/ s11036-021-01814-5

6. Cheng Y (2021) 5G Mobile Virtual Reality Optimization Solution for Communication and Computing Integration. Mobile Netw Appl. https://doi.org/10.1007/s11036-021-01812-7

Publisher's Note Springer Nature remains neutral with regard to jurisdictional claims in published maps and institutional affiliations. 